\title{
Role of tolvaptan in the management of hyponatremia in patients with lung and other cancers: current data and future perspectives
}

This article was published in the following Dove Press journal:

Cancer Management and Research

22 August 2016

Number of times this article has been viewed

\author{
Bijin Thajudeen' \\ Abdulla K Salahudeen ${ }^{1,2}$ \\ 'Department of Nephrology, Banner \\ University of Arizona Medical Center, \\ ${ }^{2}$ Department of Nephrology, Southern \\ Arizona Veterans Health Care System, \\ Tucson, AZ, USA
}

\begin{abstract}
Hyponatremia is the most frequently observed electrolyte abnormality in clinical practice, and its frequency is almost double in hospitalized cancer patients. As a subset of cancer, hyponatremia is quite common in lung cancer patients, and it is often coupled with the diagnosis of syndrome of inappropriate antidiuretic hormone secretion. The presence of hyponatremia is consequential in that its presence adversely affects cancer patients' prognosis and outcomes. Limited data suggest that correcting hyponatremia in lung cancer patients can increase response to anticancer treatment, may help reduce length of hospital stay and cost, and reduce morbidity and mortality. The type of treatment for hyponatremia depends on several factors; the key factors are the duration and severity of neurological symptoms of hyponatremia and the status of extracellular volume. When hyponatremia is caused by syndrome of inappropriate antidiuretic hormone, hypertonic saline is indicated for acute symptomatic cases, whereas fluid restriction is recommended in chronic asymptomatic hyponatremia. The latter allows a slower rate of correction, thus avoiding the dreaded complication of osmotic demyelination syndrome. Fluid restriction is, however, insufficient or impractical, and often the use of pharmacological therapy such as antidiuretic hormone receptor antagonists becomes necessary. Availability of these antagonists as an effective treatment in the management of hyponatremia has been a major breakthrough, and furthermore, its clinical or investigational use in cancer-related hyponatremia may offer a potential opportunity to gain further insights into the prognostic impact of hyponatremia correction on cancer patients' outcomes. Tolvaptan is a prototype of ADH receptor antagonists that acts at renal tubular levels to increase free water excretion without inducing major systemic electrolyte abnormalities such as hypokalemia or alkalosis. The aim of this paper is to provide a brief review while focusing on cancer hyponatremia; (1) of the epidemiology of hyponatremia and its pathophysiology and diagnostic approaches and (2) of the pharmacokinetics of tolvaptan and its clinical efficacy, safety, and compliance.
\end{abstract}

Keywords: treatment, pharmacology, safety, tolerability, efficacy, adherence

\section{Introduction}

Hyponatremia is the most common electrolyte abnormality in clinical practice, and by definition, it is considered as a disorder when serum sodium drops below $136 \mathrm{meq} / \mathrm{L} .{ }^{1}$ It occurs in $\sim 20 \%$ of hospitalized patients, and in a recent survey, its frequency was reported to be nearly double in hospitalized cancer patients. ${ }^{2}$ Hyponatremia is associated with poor clinical outcomes such as reduced survival, prolonged hospital stay, and increased hospital costs. ${ }^{3,4}$ In the United States the direct costs of treating hyponatremia annually are estimated to range from $\$ 1.6$ to $\$ 3.6$ billion, of which $70 \%$ is attributed mostly to the cost of hospitalization. ${ }^{2}$ It is also reported to be an independent predictor
Department of Nephrology University of Arizona Medical Center, I50I N Campbell Ave, Tucson, AZ 85724, USA

$\mathrm{Tel}+\mathrm{I} 5202042130$

Fax +I 5206262024

Email bijint@gmail.com 
of death in patients with cancer, heart disease, cirrhosis, or neurologic disorders. ${ }^{5}$ Furthermore, hyponatremia is also demonstrated to be associated with significant comorbidities such as gait instability and bone loss with increased fracture risks. ${ }^{6}$

\section{Hyponatremia in cancer patients}

There is a wide variation in the reported incidence and prevalence of hyponatremia in cancer patients. Various clinical studies have showed hyponatremia ranging between $25 \%$ and $44 \%$ when higher serum sodium cutoff, that is, $<136 \mathrm{meq} / \mathrm{L}$, was used, whereas rates were lower at $\sim 15 \%$ when $<130$ meq $/ \mathrm{L}$ cutoff was used. ${ }^{7}$ A recent survey conducted in 3,357 cancer patients admitted to a comprehensive cancer center reported a much higher rate of hyponatremia; using the higher sodium cutoff of $<136 \mathrm{meq} / \mathrm{L}, 47 \%$ of hospitalized cancer patients were noted to have hyponatremia, which was mild (130$135 \mathrm{meq} / \mathrm{L})$ in $36 \%$, moderate (125-130 meq/L) in $10 \%$, and severe $(<125 \mathrm{meq} / \mathrm{L})$ in $1 \% .{ }^{8}$ In this study, hyponatremia was acquired in $24 \%$ during the hospital stay. ${ }^{8}$

Hyponatremia is common in lung cancer patients, ranging between $11 \%$ and $15 \%$, and the common cause is syndrome of inappropriate release of antidiuretic hormone (SIADH). ${ }^{9,10}$ SIADH can be due to nonosmotic release of antidiuretic hormone $(\mathrm{ADH})$ from the tumor itself or due to the effect of drugs used in the treatment and palliation of cancer, like vincristine, vinblastine, and cyclophosphamide, among others. ${ }^{11}$ Some of these agents like cyclophosphamide can potentiate the actions of ADH at the renal tubular level. Similarly, opioid analgesics used for pain control in cancer patients, antidepressants used in treatment of depression in cancer patients (including tricyclics and selective serotonin reuptake inhibitors), and phenothiazines used as antiemetic agents can all stimulate ADH secretion. ${ }^{11}$ Cancer patients can develop nausea and vomiting as part of the disease or following chemotherapy which in turn can cause inappropriate ADH secretion leading to hyponatremia. There are non-ADH-related etiologies described as causes of hyponatremia in cancer patients. Patients undergoing chemotherapy receive large amounts of intravenous fluids, and hence, dilutional hyponatremia has been reported with hydration protocols using hypotonic solution. A hypervolemic hyponatremia can be induced as a result of congestive heart failure that complicates the use of antineoplastic therapy agents (eg, anthracyclines). ${ }^{12}$ It can be seen in patients who develop nephrotic syndrome due to membranous nephropathy or minimal change disease as part of the paraneoplastic syndromes. ${ }^{13}$ Hyponatremia can also be precipitated from fluid losses due to emesis or diarrhea secondary to antineoplastic therapy. Other causes of hyponatremia include adrenal insufficiency from metastasis in adrenal glands, cerebral salt wasting resulting from brain metastases, and salt wasting secondary to drugs like cisplatin. $^{14,15}$

Hyponatremia is a major cause of morbidity and mortality in cancer patients which has been shown to be an independent prognostic marker. ${ }^{3,16}$ The prognostic value of hyponatremia can vary based on several factors including the extent of the disease and pretreatment for hyponatremia. Even in patients with extensive disease, correction of sodium and amelioration of the associated clinical symptoms may help to improve the prognosis. Similarly, failure to normalize serum sodium following initiation of chemotherapy is found to be a negative prognostic factor. ${ }^{17}$ Hyponatremia can affect the performance status of patients, and this has been consistently demonstrated in various studies. ${ }^{9,18,19}$ A classic example is the relation between hyponatremia and Eastern Cooperative Oncology Group (ECOG) score which is correlated with the duration of the patient survival, response to treatment, quality of life, and comorbidity. ${ }^{19}$ ECOG score at admission showed a negative correlation with the serum sodium status. ${ }^{19}$ Treatment of hyponatremia in a small study led to a rapid correction and stabilization of the plasma sodium level which enabled the initiation of chemotherapy and subsequent amelioration of the ECOG performance status. ${ }^{9}$ Hyponatremia can also interfere with successful treatment of cancer. This is mainly because of the relationship between low Karnofsky score and hyponatremia. Patients with low Karnofsky score usually do not qualify for chemotherapy, in general. Data show that up to $40 \%$ of the patients may not receive effective primary chemotherapy due to low Karnofsky index. ${ }^{9}$ Such delay in the initiation of systemic chemotherapy can impact the outcome of treatment.

\section{Role of ADH in hyponatremia}

ADH plays a central role in water balance, and consequently in hyponatremia. ADH hormone is synthesized in the neurohypophyseal system by the cell bodies of the supraoptic nuclei. ${ }^{20}$ It is stored in the posterior pituitary and is released in response to changes in osmolality that are detected by the osmoreceptors located in the hypothalamus or by the changes in blood volume or pressure detected by baroreceptors located in the carotid sinus, aortic arch, atria, and pulmonary venous system. An increase in circulating angiotensin level also increases the secretion of ADH. There are mainly two types of receptors: V1 and V2 (Table 1). The physiological effects caused by the activation of $\mathrm{V} 1$ receptors include 
Table I Subtypes of vasopressin receptors, location, and function

\begin{tabular}{lll}
\hline Receptor & Location & Function \\
\hline VIA & Vascular smooth muscle, platelet, hepatocyte, myometrium & Vasoconstriction, platelet aggregation, myocardial hypertrophy \\
VIB & Anterior pituitary & Release of ACTH, prolactin, and endorphins \\
V2 & Collecting duct of kidney, vascular endothelium, and vascular & Insertion of AQP2 channels into apical membrane of collecting \\
& smooth muscle cell & duct, release of von Willebrand factor, and factor VIII \\
\hline
\end{tabular}

Abbreviation: ACTH, adrenocorticotropic hormone.

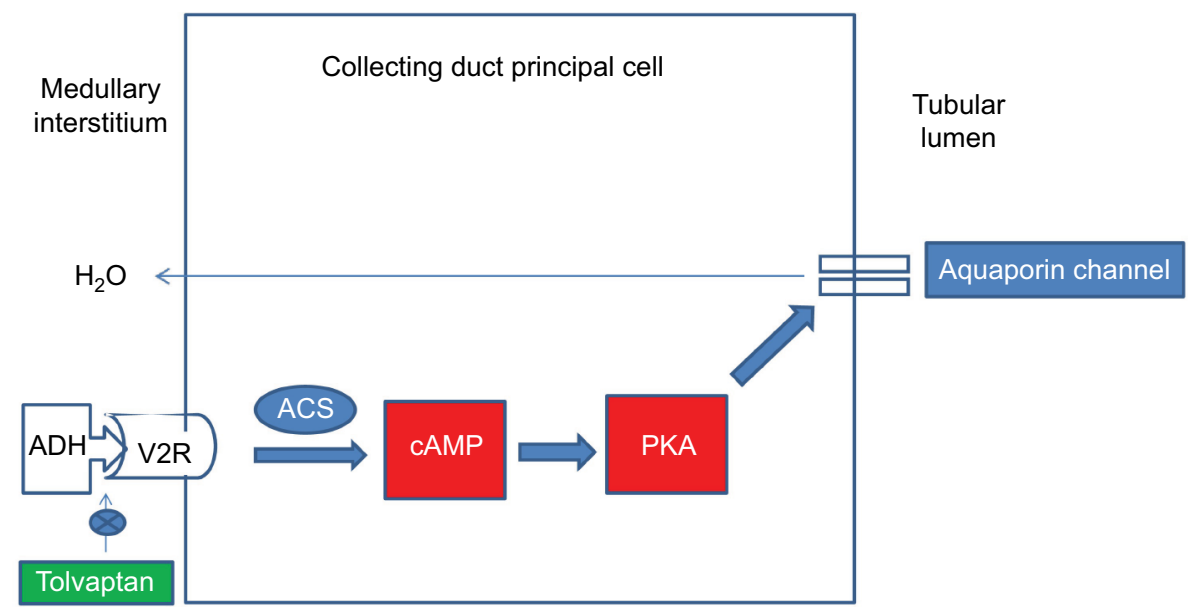

Figure I Mechanism of action of ADH and site of action of tolvaptan.

Abbreviations: $A C S$, adenyl cyclase system; $A D H$, antidiuretic hormone; PKA, protein kinase $A$; V2R, V2 receptor.

vasoconstriction, platelet aggregation, myocardial hypertrophy, and pituitary adrenocorticotropic hormone (ACTH) secretion. V2 receptor (V2R) is mainly found in the kidneys and is primarily located on the principal cells of the renal collecting tubules and the vascular endothelium, where the receptors mediate the antidiuretic effects of ADH. Binding of ADH to the V2R activates the Gs-coupled adenylyl cyclase system, which increases intracellular levels of cyclic adenosine monophosphate. This in turn activates protein kinase A, which then phosphorylates preformed AQP2 water channels localized in intracellular vesicles. This phosphorylation stimulates trafficking of the vesicles to the apical membrane, followed by insertion of water channel AQP2 into the membrane which increases water permeability (Figure 1). ${ }^{21-23}$ Additionally ADH can increase thirst and thereby increase water intake, which helps normalize plasma osmolality.

\section{SIADH and lung cancer}

SIADH is a syndrome associated with inappropriate secretion of ADH in the absence of plasma hypotonicity. SIADH, first described in lung cancer patients, is most commonly found in small cell lung cancer (SCLC). ${ }^{9,10}$ SIADH is also seen in $2 \%-4 \%$ of non-small cell lung cancer patients. ${ }^{9}{ }^{90}$ In SIADH, $\mathrm{ADH}$ secretion is persistently elevated regardless of the tonicity, maintained by the nonosmotic factors primarily due to the ectopic production of ADH. The first clinical case of a patient with SIADH was presented by Schwartz et al who described two patients with lung cancer and hyponatremia that was attributed to ectopic secretion of ADH by tumor cells. ${ }^{24}$ Secretion of ADH by cancer cells was initially demonstrated by Klein et $\mathrm{al}^{25}$ and George et al. ${ }^{26}$ Presence of SIADH has been associated with higher propensity for central nervous system metastases, poor response to chemotherapy and advanced stage of cancer. ${ }^{26,27}$

The relation between SIADH and ectopic production of ADH by tumor cells has been demonstrated in in vitro studies. These studies have shown high concentration of immunereactive ADH in tumor extracts of patients with SIADH. Northern blot and $\mathrm{S}_{1}$-nuclease analysis demonstrated the production of ADH mRNA in tumors and tumor cell lines from patients with SCLC and hyponatremia. ${ }^{28}$ Similarly, ADH has also been detected in SCLC tumors and tumor cell lines by bioassay of antidiuretic activity and by radioimmunoassay. Of note is that SCLCs causing SIADH are made up of heterogeneous cell populations; some contain $\mathrm{ADH}$ and others do not. Tissue cultures derived from ADH-producing small cell carcinoma demonstrated ADH in only a fraction of cells with most cells staining negative. Evidence for the production of ADH by selected cells is also supported by the finding of persistent SIADH in patients who had marked decrease in the tumor size. ${ }^{28-31}$

\section{Symptoms of hyponatremia}

The symptoms of hyponatremia are related to both the severity of hyponatremia and the rapidity of changes in plasma sodium 
concentrations. In acute hyponatremia, symptoms are mostly due to cerebral edema from intracellular shift of water. Common symptoms of hyponatremia include headache, nausea, vomiting, muscle cramps, lethargy, disorientation, and depressed reflexes. Symptoms can vary based on the level of serum sodium. Sodium levels of $125-130 \mathrm{meq} / \mathrm{L}$ are usually associated with nausea and malaise. When the plasma sodium falls in the range 115-125 meq/L, headache, lethargy, and obtundation set in, and for levels $<115$ meq/L, seizures, coma, respiratory arrest, or brain herniation may occur. The encephalopathy of hyponatremia though reversible can lead to permanent neurologic damage or death. ${ }^{11}$ Noncardiogenic pulmonary edema has also been described. ${ }^{32}$ These symptoms of hyponatremia may predate or accompany the underlying cancer, and they may not correlate with stage and anatomical spread of cancer or response to therapy. In chronic hyponatremia, there is enough time for osmolyte movement including sodium, potassium, and chloride out of the brain cells into the cerebrospinal fluid that prevents the intracellular water movement and cerebral edema; hence, most patients are asymptomatic. . $^{33,34}$

\section{Diagnosis of hyponatremia}

Establishing the diagnostic type from three clinically distinct types, that is, euvolemic, hypovolemic, and hypervolemic hyponatremia is important as management can vary markedly, and wrong clinical diagnosis and adoption of wrong treatment can have serious consequences. An accurate diagnosis can be made based on clinical history, physical examination, and laboratory tests. The first step in assessment of any hyponatremia is the measurement of serum osmolality. ${ }^{35}$ A normal serum osmolality would suggest the presence of pseudo hyponatremia, which is seen in patients with hyperglobulinemia and hypertriglyceridemia. ${ }^{1}$ Once true hyponatremia is confirm, the next step is the assessment of volume status. ${ }^{36}$ Clinical examination as well as laboratory tests confirm signs of volume depletion (orthostatic changes in heart rate and blood pressure, evidence of hemoconcentration, a blood urea nitrogen to creatinine ratio $>40$, low urine sodium and chloride concentrations $<20 \mathrm{meq} / \mathrm{L}$, and have fractional excretion of these electrolytes $<1 \%$ ) and help in the exclusion of hypovolemic hyponatremia (emesis, cerebral salt wasting, and mineralocorticoid deficiency). Presence of adrenal insufficiency can be associated with low serum cortisol levels, presence of adrenal metastasis in imaging hyperkalemia, eosinophilia, and skin pigmentation changes. ${ }^{12}$ Patients with subcutaneous edema and ascites are deemed hypervolemic and differential diagnosis supporting hypervolemic hyponatremia should be considered. If the patient is deemed euvolemic, the next step is assessment of urine osmolality to assess whether the ability of kidney to dilute urine is intact. Since a normal kidney can excrete up to 20-30 L of water per day, it is unusual for patients to get hyponatremia unless their water intake is far more than the amount that can be handled by the kidney (as happens in polydipsia). ${ }^{1}$ Hence in the presence of hyponatremia and euvolemia, kidneys will try to maximally dilute the urine in an effort to get rid of the excess water which will result in a urine osmolality $<100 \mathrm{mom} / \mathrm{L} .{ }^{37}$ In hyponatremic patients with inappropriately concentrated urine $(>200 \mathrm{mOsm} / \mathrm{L})$, SIADH is suspected. ${ }^{33}$ Euvolemic hyponatremia seen in SIADH can also be seen in patients with hypothyroidism or glucocorticoid deficiency. Assessment of thyroid and adrenal gland functions will help to exclude these causes. ${ }^{38}$

\section{Tolvaptan in the treatment of hyponatremia in lung cancer patients}

Recognition and withdrawal of inciting agents causing hyponatremia is an important first step. In hypovolemic hyponatremia, isotonic saline corrects the hyponatremia, and thus, asymptomatic hyponatremia due to extracellular fluid volume depletion should be treated with isotonic saline infusion. In the case of cisplatin-induced renal salt wasting, cisplatin should be temporarily withdrawn until there is recovery of renal and tubular dysfunction. Treatment of SIADH includes treatment of the underlying cancer with chemotherapy and removal of any SIADH causing drugs.

In addition to the correction of underlying causes, additional treatment options for the correction of asymptomatic hyponatremia of SIADH include fluid restriction and the use of pharmacological agents. Fluid restriction is generally ineffective, often requiring the use of pharmacological agents. These pharmacological agents can enhance free water excretion either by increasing solute excretion or interfering with medullary concentration gradient or by directly blocking the ADH receptors. Methods to increase solute excretion include increasing protein intake and use of urea or salt tablets. Use of urea is limited by unpleasant taste and potential liver and renal toxicity. Loop diuretics are the major agents that are used to block medullary concentration gradient. They are effective in instances where there are high levels of $\mathrm{ADH}$, reflected by high urine osmolality (especially urine osmolality $>600 \mathrm{mOsm} / \mathrm{L}$ ). However, use of diuretics can be associated with major side effects such as electrolyte imbalance and precipitation of volume depletion. ${ }^{39}$ Demeclocycline, a tetracycline analog that negates the effects of ADH at the level of renal tubules, is seldom used due to its slow onset of action, hepatic as well as renal toxicity. ${ }^{40}$ 
An effective and sensible way of blocking of ADH receptors is by using recently available $\mathrm{ADH}$ receptor antagonists. They are otherwise called aquaretics to distinguish them from the renal effects produced by classical diuretic agents that are natriuretic and kaliuretic. ${ }^{41}$ Clinical studies using ADH receptor antagonists showed a reduction in urine osmolality, thus consequently raising serum sodium concentrations. The diuretic effect is independent of any increase in urinary excretions of sodium, potassium, or chloride..$^{42,43}$ Out of all the $\mathrm{ADH}$ receptor antagonists, the most commonly used one is tolvaptan.

\section{Review of pharmacology, mode of action, pharmacokinetics of tolvaptan}

Tolvaptan is a selective orally administered V2R antagonist. It has a half-life of 6-8 hours. It binds reversibly (98\%) to plasma proteins. It is extensively metabolized by the liver, and $<1 \%$ of intact active substance is excreted unchanged in the urine. It follows a linear pharmacokinetics for doses of $15-60 \mathrm{mg}$. The starting dose of tolvaptan is $15 \mathrm{mg}$ on the first day, and the dose can be titrated to 30 and $60 \mathrm{mg}$ at 24-hour intervals if the serum sodium remains $<135 \mathrm{~mol} / \mathrm{L}$ or the increase in serum sodium has been $<5 \mathrm{mmol} / \mathrm{L}$ in the previous 24 hours. ${ }^{44}$ Maximum recommended dose of treatment of hyponatremia is $60 \mathrm{mg}$ daily ${ }^{38}$ During the course of treatment, water restriction should be avoided to prevent dehydration. In case of inadequate improvement in serum sodium levels, other treatment options should be considered, either in place of or in addition to tolvaptan, keeping in mind that combination therapy might increase the risk of overly rapid correction of serum sodium. Once there is an appropriate rise in serum sodium levels, the status of underlying disease should be evaluated to assess the need for continuation of tolvaptan.

Monitoring response is based on the improvement in serum sodium. Monitoring of ADH levels can be useful in some cases since ADH levels can decrease with successful treatment of cancer. ${ }^{29}$ Urine osmolality can act as the surrogate marker for $\mathrm{ADH}$ effect. A reduction in urine osmolality compared to baseline can help in determining the response to therapy. ${ }^{39}$ Response can also be monitored by assessment of electrolyte-free water clearance. ${ }^{45}$ In SIADH, electrolyte-free water clearance is negative, and therefore, limited excretion of water is expected. With treatment using tolvaptan, there will be substantial improvement in electrolyte-free water clearance. Similarly uric acid can also be used as a marker of correction. With resolution of SIADH, uric acid levels will increase ${ }^{29}$ Fractional excretion of uric acid can also be used for monitoring. Fractional excretion of uric acid that will be high in SIADH will reduce following the correction of SIADH. ${ }^{29,46}$

\section{Safety and tolerability of tolvaptan}

Common side effects of tolvaptan include dry mouth, thirst, pollakiuria, increased urinary frequency, and rarely dizziness, nausea, and orthostatic hypotension. ${ }^{47}$ Other less common side effects include hypoglycemia, hyperuricemia, syncope, dizziness, headache, malaise, and diarrhea. There are rare reports of hypernatremia and polyuria. Hepatotoxicity with tolvaptan is a major concern based on the TEMPO trial (this trial examined the effect of tolvaptan on the progression of polycystic kidney disease). In the TEMPO trial, an increased incidence of clinically significant elevations of alanine aminotransferase was observed in patients treated with tolvaptan compared to those receiving placebo, and most of the liver enzyme abnormalities were observed during the first 18 months of treatment. ${ }^{48}$ The elevations gradually improved once tolvaptan was discontinued supporting the view that tolvaptan has the potential to cause potentially irreversible and fatal liver injury. ${ }^{48}$ It is recommended to check liver function tests in patients taking tolvaptan who report symptoms indicating liver injury like fatigue, anorexia, right upper abdominal discomfort, dark urine, or jaundice. ${ }^{45}$ If liver injury is suspected, tolvaptan should be promptly discontinued and appropriate treatment should be instituted..$^{45}$ Tolvaptan should not be re-initiated in these patients unless it is proven that the cause for the observed liver injury is unrelated to treatment with tolvaptan. Another major side effect is the rapid correction of hyponatremia, which in turn may lead to the development of the osmotic demyelination syndrome. Osmotic demyelination syndrome is a disorder characterized by dysarthria, mutism, dysphagia, lethargy, affective changes, spastic quadriparesis, seizures, coma, or death. It can develop 1 to several days after aggressive treatment of hyponatremia by any method, including water restriction alone. The underlying pathology is shrinkage of the brain, disruption of the blood-brain barrier, influx of immune-competent proteins, and subsequent demyelination of pontine and extra pontine neurons. ${ }^{49}$

Close monitoring of sodium is also important while using tolvaptan for the treatment of hyponatremia. This is due to the fact that most cases of SIADH from lung cancer might resolve following successful chemotherapy and continued use of tolvaptan can lead to overcorrection..$^{10}$ Hence, it is recommended to start tolvaptan therapy only when patients 
are in the hospital. This allows for close monitoring of the therapeutic response and avoids overcorrection of sodium. Once initiated on tolvaptan, sodium should be monitored at frequent intervals. Generally, measurement of serum sodium is recommended every 4-6 hours, but the frequency should be increased if patients are at higher risk for osmotic demyelination because of alcoholism, malnutrition, hypokalemia, and serum sodium $<120 \mathrm{meq} / \mathrm{L} .{ }^{49}$ One additional factor that helps to avoid overly rapid correction with tolvaptan is the recommendation that fluid restriction should not be used during the active phase of correction, thereby allowing the patient's thirst to compensate for an overly vigorous aquaresis. In instances where there is rapid correction, tolvaptan can be held or dose can be reduced subsequently, and sodium can be re-lowered using hypotonic solution (via oral and/or intravenous routes) with or without desmopressin. This will induce a prompt decline in the serum sodium concentration with an acceptable final gradient of correction. Although there are reports of osmotic demyelination syndrome with the use of tolvaptan, most cases have been due to inappropriate treatment strategies. Patients may also need constant volume monitoring since tolvaptan treatment may result in severe dehydration, which constitutes a risk factor for renal dysfunction. If dehydration becomes evident, dose of tolvaptan should be reduced and fluid intake should be increased.

Tolvaptan is metabolized by the cytochrome P450 isoenzyme CYP3A4, which is involved in the metabolism of a large number of common drugs, resulting in a high risk of pharmacokinetic interactions. Caution should be exercised in using tolvaptan along with CYP3A4 inhibitors like ketoconazole, macrolide antibiotics, and diltiazem (which can increase plasma concentration) or CYP3A4 inducers like rifampicin and barbiturates (which can decrease plasma concentration). Other agents that can have significant interaction with tolvaptan include digoxin, DDAVP, and grapefruit juice. ${ }^{19,21,47,50}$

Although tolvaptan does not need dose adjustment in elderly patients, its safety and efficacy in children and adolescents aged $<18$ years have not yet been established, and hence, tolvaptan is not recommended in the pediatric age group. There is no dose adjustment based on sex or race. Although tolvaptan is not contraindicated with decreased renal function, it may not be effective if the serum creatinine is $>2.5 \mathrm{mg} / \mathrm{dL} .{ }^{44}$ Women of childbearing potential should use adequate contraceptive measures during tolvaptan use. Tolvaptan is contraindicated in patients with hypersensitivity to the active substance/excipients of tolvaptan formulation or patients with anuria, hypovolemic hyponatremia, pregnant or breast feeding females, and patients with impaired thirst, decreased access to free water or altered mental status.

Tolvaptan is also not recommended in patients with moderate to severe symptoms of hyponatremia including seizure and coma. Use of tolvaptan in such instances can lead to potentially rapid correction, placing the patients at risk for neurological complications. Three percent saline should be the first-line therapy in such instances since these patients require predictable and titratable correction. ${ }^{51}$

Ideally, tolvaptan should be continued until the underlying disease is adequately treated or until hyponatremia is no longer a clinical problem. ${ }^{45}$ Although tolvaptan has a number of benefits in patients with lung cancer, US Food and Drug Administration recommendations (http://www. fda.gov/Safety/MedWatch/SafetyInformation/SafetyAlertsforHumanMedicalProducts/ucm350185.htm) arising from concerns of potential liver toxicity with chronic use, clinicians are required to limit its use to no more than 30 days. It is not clear how tolvaptan should be withdrawn in patients receiving it for chronic hyponatremia. Factors that would be of consideration are the underlying cause of hyponatremia, effectiveness of other treatment options, and tolvaptan dosage. For patients on a 15-mg dose, tolvaptan can be withdrawn abruptly or reduced gradually/taken on alternate days, as clinically indicated. If patients are on higher doses, that is, $30 \mathrm{mg}$, gradual dose reduction is preferred until the dose reaches $15 \mathrm{mg}$ and then it can be withdrawn.

\section{Efficacy studies on tolvaptan Studies mostly in noncancer patients}

Although there have been a number of clinical trials using tolvaptan, many of them did not highlight the number of lung cancer patients involved or their outcomes. Hence there is a scarcity of lung cancer-specific information for tolvaptan. The largest trials in this category are the SALT trials. SALT trials (SALT-1 and SALT-2) were double-blind, placebo-controlled, clinical trials in mostly noncancer medical patients, which compared the effect of tolvaptan to placebo in correcting the hyponatremia. A total of 424 patients with euvolemic or hypervolemic hyponatremia (serum sodium $<135 \mathrm{meq} / \mathrm{L}$ ) due to various underlying causes (heart failure, liver cirrhosis, SIADH, and others) were treated for 30 days with tolvaptan $(n=216)$ or placebo $(n=208)$. After the initial dose of $15 \mathrm{mg}$, doses were gradually increased to 30 and $60 \mathrm{mg} /$ day depending on response using a 3-day titration scheme. The mean serum sodium concentration at trial entry was $129 \mathrm{meq} / \mathrm{L}$ (range 114-136 meq/L). The primary endpoint for these trials was the change in serum sodium from baseline to day 4 and 
baseline to day 30 . At day 4 and day 30 of treatment with tolvaptan, sodium increased significantly than placebo in these patients ( $40 \%$ vs $13 \%$ in the SALT- 1 trial and $55 \%$ vs $11 \%$ in the SALT- 2 trial). None of the patients were on fluid restriction. Tolvaptan was found to be superior to placebo with respect to several parameters; change in the average daily area under the curve for serum sodium levels from baseline to day 4 and from baseline to day 30 , the mean serum sodium concentration at each visit, the time to normalized serum sodium concentrations, the percentage of patients with serum sodium concentrations that were normal on day 4 and on day 30 , and the categorical change in the serum sodium concentration from baseline to day 4 and from baseline to day 30. Tolvaptan maintained its superiority over placebo from initial observation point ( 8 hours after administration of the first dose) to the last day of treatment (day 30) in patients with all levels of hyponatremia irrespective of the underlying cause. Overcorrection of sodium was seen only in four of 223 patients $(1.8 \%)$ on day 1 . Similarly only four of 223 patients $(1.8 \%$ ) had a serum sodium level 146 meq/L or above at some point during the study period. Except for thirst (14\% vs $5 \%)$, dry mouth (13\% vs $4 \%$ ), and increased urination ( $7 \%$ vs $3 \%)$, tolvaptan was well tolerated by most of the patients. Sodium levels trended down to levels comparable to that of placebo patients after a week of stopping tolvaptan. A subgroup analysis of SIADH patients in the SALT trials (out of the total 448 patients included in the SALT trials, 110 had a primary diagnosis of SIADH) also showed improvement in sodium with tolvaptan therapy. Only $5.9 \%$ of patients treated with tolvaptan exceeded the treatment target sodium limits, and there were no reports of neurological symptoms suggestive of osmotic demyelination syndrome. ${ }^{52}$

Another trial that looked at the long-term effects of tolvaptan was the SALTWATER trial, an open label extension of SALT-1 and SALT-2 trials. It enrolled 111 patients, and the mean follow-up was 701 days. There was prompt improvement in sodium with use of tolvaptan as early as day 1 with subsequent normalization of serum sodium and normal values were maintained for the entire study periods. Mean serum sodium increased from $130.8 \mathrm{mmol} / \mathrm{L}$ at baseline to $>135 \mathrm{mmol} / \mathrm{L}$ throughout the observation period. Rapid correction of sodium was rarely observed in this study as well. When treatment was discontinued, serum sodium concentrations decreased to approximately baseline values (at day 7), despite the reinstatement of standard care therapy, indicating a need for continued tolvaptan therapy to maintain serum sodium. Although a majority of the patients in this study had SIADH (52.3\%), the number of patients who had a diagnosis of cancer is not known. ${ }^{53}$
Verbalis et al looked at the effects of tolvaptan on neurocognitive function in 57 patients (aged $\geq 50$ years) with chronic asymptomatic euvolemic or hypervolemic hyponatremia. ${ }^{41}$ Patients were randomly assigned 1:1 to receive tolvaptan or matching placebo. Mean serum sodium concentration at entry was 120-135 meq/L. Primary outcome measured was the change on the neurocognitive composite score, and secondary outcomes measured included individual neurocognitive domain scores, overall neurocognitive composite score, gait and postural stability test results, and serum sodium concentrations. At the end of the study period ( 3 weeks), there was no difference in overall neurocognitive composite scores between groups, except for the psychomotor speed domain, which improved significantly following hyponatremia correction with tolvaptan (treatment effect, $0.27 ; 95 \%$ confidence interval, $0.04-0.51 ; P<0.03)$. There was also improvement in the mean serum sodium concentration (129-136 meq/L in the tolvaptan group and from 130-132 meq/L in the placebo group). Major limitations for this study included limited number of patients and short follow-up. ${ }^{41}$

\section{Studies in cancer patients}

Salahudeen et $\mathrm{al}^{54}$ conducted a double-blind randomized controlled study of tolvaptan versus placebo in hyponatremic cancer patients, irrespective of the cancer type, using an adaptive design (17 in tolvaptan group and 13 in placebo group). The primary objective of the study of determining superiority of tolvaptan over placebo was deemed met by the Safety and Data Monitoring Board, requiring the study to be stopped early. A majority of the cancers were solid tumors $(69 \%$ in placebo and $65 \%$ in tolvaptan group; total of seven lung cancer patients). Starting dose was $15 \mathrm{mg}$, and the dose was gradually titrated up according to response to achieve a correction of $6-12 \mathrm{meq} / \mathrm{L}$ targeting sodium of $>136 \mathrm{meq} / \mathrm{L}$. The mean serum sodium at entry was comparable between the groups. The primary endpoint of hyponatremia correction was met by 16 of 17 patients who received tolvaptan and by one of 13 patients who received placebo $(94 \%$ vs $8 \% ; P<0.001)$ at day 14. The secondary endpoints between the tolvaptan and placebo groups for length of stay and change in the Mini-Mental State Examination score were not significantly different. There were no cases of overcorrection. Major side effects reported included dry mouth, polydipsia, and polyuria, leading to withdrawal of $13 \%$ of the study subjects. ${ }^{8,54}$

\section{Studies in lung cancer patients}

Petereit et al looked at ten patients with advanced SCLC and severe SIADH. ${ }^{9}$ All the patients were monitored for clinical improvement based on ECOG status, commencement of 
chemotherapy, and correction of sodium levels. Patients received tolvaptan at a dose of $15 \mathrm{mg}$ for a median of 4 days. There was significant clinical improvement in sodium in the study participants. Moreover, use of tolvaptan significantly improved ECOG performance status which subsequently allowed initiation of chemotherapy especially with platinum agents. It also allowed for less hospital days and avoided emergent hospital visits. There were no serious adverse events reported during the study. The median duration of sodium maintenance was 17.5 (range: 2-614) days after tolvaptan discontinuation. ${ }^{9}$

A similar study done by Kenz et al $^{55}$ followed 13 patients with paraneoplastic SIADH out of which seven had SCLC. Serum sodium levels normalized in the majority of the patients (11/13 patients), at a dose of $7.5 \mathrm{mg}$ tolvaptan on alternate days, despite stopping fluid restriction. ${ }^{55}$

\section{Summary of the efficacy studies}

Although limited data are available in lung cancer patients, all the aforementioned studies in cancer and noncancer patients showed a consistent benefit with the use of tolvaptan in normalizing sodium. It meets the requirements of rapidity, efficacy, and safety based on these studies. But the effect was not sustained once tolvaptan was discontinued reiterating the fact that chronic tolvaptan therapy is mandated in most of the patients with SIADH especially in the presence of lung cancer. The side effect profile of the drug also seems to be acceptable.

\section{Tolvaptan impact on patients' quality of life, satisfaction/ acceptability, and adherence}

Use of tolvaptan in lung cancer patients has multiple benefits - some at least in theory - which can impact the quality of life: 1) patients can undergo chemotherapy without concerns for further aggravation of hyponatremia, 2) in patients not treated with chemotherapy, these agents may reduce the symptoms of hyponatremia, 3) reduces hospitalization due to hyponatremia and symptoms related to hyponatremia, 4) treatment of hyponatremia without having to restrict oral fluids (this is important in patients undergoing chemotherapy with potentially nephrotoxic agents, eg, cisplatin), 5) it can facilitate the administration of chemotherapy cycles in a timely manner, which in turn can improve the chance of patient's prognosis and survival, 6) V2R antagonist therapy may permit early hospital discharge with its innumerable benefits, 7) decreases the number of emergency visits, prevents hospital readmission, and reduces possible admission to intensive care units, and last but not least, 8) the negative water balance induced by aquaretic agents has less adverse effect on neurohormonal activation and renal function than comparable amounts of urine output induced by loop diuretic agents. ${ }^{8,56,57}$ Over and above, tolvaptan is preferred in patients where the treatment of the malignancy that is causing the SIADH requires an immediate start of chemotherapy that cannot be administered without adequate hydration volumes, or in malnourished patients, for whom liquid restriction would worsen their nutritional state. Some of these observations were substantiated in the clinical trials discussed earlier. In the SALT trials, the results of selfassessed health status using the SF-12 Health Survey for the mental scores showed statistically significant and clinically relevant improvements for tolvaptan treatment compared to placebo. SALT trial analysis also showed that use of tolvaptan nominally shortened hospital stay in the tolvaptan-treated patients, and this difference achieved statistical significance when the severe hyponatremia patients with SIADH were considered. ${ }^{58}$ In the subgroup analysis of SIADH patients in the SALT trial, tolvaptan, in addition to the efficacy in increasing serum sodium also had impact on the mental and physical health of patients (physical health composite score and mental health composite score) assessed using the SF-12 General Health Survey. Additionally, the use of tolvaptan has been recommended as a safe treatment option for terminally ill patients considering palliative care. Even in cancer patients on palliative care, and in patients with persistent hyponatremia, to improve symptoms, tolvaptan can be continued. As the long-term results for patients with SCLC are still poor, treatment of hyponatremia by itself might improve the overall outcome of the cancer.

Clinical trials have consistently shown reasonably good adherence to tolvaptan therapy, especially short-term. In the subgroup analysis of SIADH patients in the SALT trials, there were 52 patients who were assigned to the tolvaptan arm. Of these, 42 completed the 30-day follow-up with $\sim 80 \%$ adherence. Ten patients discontinued the treatment and half of them discontinued due to adverse events. It is also notable that only four patients required fluid restriction, which is significant in many patients with SIADH who find it difficult to adhere to water restriction. The nonrequirement of fluid restriction in the presence of tolvaptan can improve patient adherence and compliance with tolvaptan therapy. The other factors that might have contributed to a high degree of compliance include lower number of hospital days and improvement in physical as well as mental health composite scores following the treatment. ${ }^{51}$ Similarly, in the study by Salahudeen et al, ${ }^{54}$ the compliance rate was $70 \%$. Out of the 24 patients assigned 
to tolvaptan, 17 patients completed the study. Out of the seven patients in whom the therapy was terminated before the end of study, three patients terminated because of side effects. The mean number of days in hospital was 5 days less than for the placebo group ( $26 \pm 15$ days vs $21 \pm 15$ days). ${ }^{8}$ In the study by Verbalis et $\mathrm{al}^{41}$ a total of 29 patients were placed on tolvaptan arm and 26 patients completed study with compliance rate of $90 \%$. Two patients withdrew from the study due to adverse events. ${ }^{41}$ On the other hand, in the open label extension of SALT trials that followed up patients for nearly 2 years, the compliance rate was only $42 \%$. Out of the 111 patients enrolled in the trial, only 47 patients completed the study. Out of the 64 patients who did not complete the study, 19 withdrawals were due to adverse events and eleven were due to deaths from adverse events. Overall, short-term studies showed $70 \%-90 \%$ compliance and acceptability, whereas on long-term follow-up the compliance was only $42 \%$.

\section{Conclusion}

Hyponatremia is a common electrolyte disorder in cancer patients particularly in lung cancer patients, which complicates cancer management and its outcomes. In cancer, SIADH may be due to ectopic $\mathrm{ADH}$ production by tumor cells or due to stimulation of ADH secretion or potentiation of AVP effects by anticancer drugs or palliative medications. Studies have shown that treating hyponatremia in lung cancer patients with V2R antagonist tolvaptan can improve hyponatremia and the ECOG score making chemotherapy effective and amenable to more patients. Clinical and laboratory assessments play the key role in distinguishing SIADH from other causes. Symptomatic patients with hyponatremia are treated initially with hypertonic saline, whereas asymptomatic patients are generally managed with fluid restriction or pharmacological agents. The former is less effective in cancer patients as a high fluid intake is required for most of the patients. ADH receptor antagonists, especially tolvaptan seem to be an effective treatment strategy for hyponatremia. In the cancer patients, the use of $\mathrm{ADH}$ receptor antagonist could improve hyponatremic symptoms even in patients on palliative care. However, at the same time the use of tolvaptan is restricted by the US Food and Drug Administration due to potential safety concerns of chronic use. More studies are required to address these concerns since tolvaptan is an excellent choice for its use in cancer hyponatremia especially in patients with lung cancer.

\section{Disclosure}

Dr Salahudeen was a consultant and his institution received grants from Otsuka for works outside of submitted manuscript.
He has also received travel support from Otsuka. The authors report no other conflicts of interest in this work.

\section{References}

1. Adrogué HJ, Madias NE. Hyponatremia. N Engl J Med. 2000;342(21): $1581-1589$.

2. Upadhyay A, Jaber BL, Madias NE. Epidemiology of hyponatremia. Semin Nephrol. 2009;29(3):227-238.

3. Gill G, Huda B, Boyd A, et al. Characteristics and mortality of severe hyponatraemia - a hospital-based study. Clin Endocrinol (Oxf). 2006; 65(2):246-249.

4. Shea AM, Hammill BG, Curtis LH, Szczech LA, Schulman KA. Medical costs of abnormal serum sodium levels. J Am Soc Nephrol. 2008;19(4):764-770.

5. Waikar SS, Mount DB, Curhan GC. Mortality after hospitalization with mild, moderate, and severe hyponatremia. Am J Med. 2009;122(9): 857-865.

6. Verbalis JG, Barsony J, Sugimura Y, et al. Hyponatremia-induced osteoporosis. J Bone Miner Res. 2010;25(3):554-563.

7. Castillo JJ, Vincent M, Justice E. Diagnosis and management of hyponatremia in cancer patients. Oncologist. 2012;17(6):756-765.

8. Doshi SM, Shah P, Lei X, Lahoti A, Salahudeen AK. Hyponatremia in hospitalized cancer patients and its impact on clinical outcomes. Am J Kidney Dis. 2012;59(2):222-228.

9. Petereit C, Zaba O, Teber I, Lüders H, Grohé C. A rapid and efficient way to manage hyponatremia in patients with SIADH and small cell lung cancer: treatment with tolvaptan. BMC Pulm Med. 2013;13:55.

10. List AF, Hainsworth JD, Davis BW, Hande KR, Greco FA, Johnson DH. The syndrome of inappropriate secretion of antidiuretic hormone (SIADH) in small-cell lung cancer. J Clin Oncol. 1986;4(8): 1191-1198.

11. Grohé C, Berardi R, Burst V. Hyponatraemia-SIADH in lung cancer diagnostic and treatment algorithms. Crit Rev Oncol Hematol. 2015;96(1):1-8

12. Sawyer DB. Anthracyclines and heart failure. N Engl J Med. 2013; 368(12):1154-1156.

13. Birkeland SA, Storm HH. Glomerulonephritis and malignancy: a population-based analysis. Kidney Int. 2003;63(2):716-721.

14. Iyer AV, Krasnow SH, Dufour DR, Arcenas AS. Sodium-wasting nephropathy caused by cisplatin in a patient with small-cell lung cancer. Clin Lung Cancer. 2003;5(3):187-189.

15. Palmer BF. Hyponatraemia in a neurosurgical patient: syndrome of inappropriate antidiuretic hormone secretion versus cerebral salt wasting. Nephrol Dial Transplant. 2000;15(2):262-268.

16. Hansen O, Sorensen P, Hansen KH. The occurrence of hyponatremia in SCLC and the influence on prognosis: a retrospective study of 453 patients treated in a single institution in a 10-year period. Lung Cancer. 2010;68(1):111-114.

17. Petereit $\mathrm{C}, \mathrm{Zaba} \mathrm{O}$, Teber I, Groh C. Ist die Hyponatriämie (HN) ein Prognosefaktor für das Gesamtüberleben der Patienten mit Lungenkarzinom? [Is hyponatremia a prognostic marker of survival for lung cancer?]. Pneumologie. 2011;65(9): 565-571.

18. Jacot W, Colinet B, Bertrand D, et al. Quality of life and comorbidity score as prognostic determinants in non-small-cell lung cancer patients. Ann Oncol. 2008;19(8):1458-1464.

19. Sengupta A, Banerjee SN, Biswas NM, et al. The incidence of hyponatraemia and its effect on the ECOG performance status among lung cancer patients. J Clin Diagn Res. 2013;7(8):1678-1682.

20. Guyton AC, Hall JE. Textbook of Medical Physiology, 10th ed. Philadelphia (PA): W.B. Saunders; 2000.

21. Bhatt PR, McNeely EB, Lin TE, Adams KF, Patterson JH. Review of tolvaptan's pharmacokinetic and pharmacodynamic properties and drug interactions. J Clin Med. 2014;3(4):1276-1290.

22. Sorensen JB, Andersen MK, Hansen HH. Syndrome of inappropriate secretion of antidiuretic hormone (SIADH) in malignant disease. J Intern Med. 1995;238(2):97-110.

23. Lee CR, Watkins ML, Patterson JH, et al. Vasopressin: a new target for the treatment of heart failure. Am Heart J. 2003;146(1):9-18. 
24. Schwartz WB, Bennett W, Curelop S, Bartter FC. A syndrome of renal sodium loss and hyponatremia probably resulting from inappropriate secretion of antidiuretic hormone. Am J Med. 1957;23(4):529-542.

25. Klein LA, Rabson AS, Worksman J. In vitro synthesis of vasopressin by lung tumor cells. Surg Forum. 1969;20:231-233.

26. George JM, Capen CC, Phillips AS. Biosynthesis of vasopressin in vitro and ultrastructure of a bronchogenic carcinoma. Patient with the syndrome of inappropriate secretion of antidiuretic hormone. J Clin Invest. 1972;51(1):141-148.

27. Chute JP, Taylor E, Williams J, Kaye F, Venzon D, Johnson BE. A metabolic study of patients with lung cancer and hyponatremia of malignancy. Clin Cancer Res. 2006;12(3 Pt 1):888-896.

28. Gross AJ, Steinberg SM, Reilly JG, et al. Atrial natriuretic factor and arginine vasopressin production in tumor cell lines from patients with lung cancer and their relationship to serum sodium. Cancer Res. 1993; 53(1):67-74.

29. Hainsworth JD, Workman R, Greco FA. Management of the syndrome of inappropriate antidiuretic hormone secretion in small cell lung cancer. Cancer. 1983;51(1):161-165.

30. Pettengill OS, Faulkner CS, Wurster-Hill DH, et al. Isolation and characterization of a hormone-producing cell line from human small cell anaplastic carcinoma of the lung. J Natl Cancer Inst. 1977;58(3):511-518.

31. Baylin SB, Weisburger WR, Eggleston JC, et al. Variable content of histaminase, L-dopa decarboxylase and calcitonin in small-cell carcinoma of the lung. Biologic and clinical implications. $N$ Engl J Med. 1978; 299(3):105-110.

32. Ayus JC, Varon J, Arieff AI. Hyponatremia, cerebral edema, and noncardiogenic pulmonary edema in marathon runners. Ann Intern Med. 2000; 132(9):711-714.

33. Palmer BF, Gates JR, Lader M. Causes and management of hyponatremia. Ann Pharmacother. 2003;37(11):1694-1702.

34. Adrogué HJ. Consequences of inadequate management of hyponatremia. Am J Nephrol. 2005;25(3):240-249.

35. Gennari FJ. Current concepts. Serum osmolality. Uses and limitations. N Engl J Med. 1984;310(2):102-105.

36. Chung HM, Kluge R, Schrier RW, Anderson RJ. Clinical assessment of extracellular fluid volume in hyponatremia. Am J Med. 1987; 83(5):905-908.

37. Hariprasad MK, Eisinger RP, Nadler IM, Padmanabhan CS, Nidus BD. Hyponatremia in psychogenic polydipsia. Arch Intern Med. 1980; 140(12):1639-1642.

38. Mali P, Muduganti SR, Mujibur R, Murali N. Tolvaptan for SIADH in myelodysplastic syndrome with blast crisis. WMJ. 2015;114(2):66-68.

39. Watanabe K, Dohi K, Sugimoto T, et al. Short-term effects of low-dose tolvaptan on hemodynamic parameters in patients with chronic heart failure. J Cardiol. 2012;60(6):462-469.

40. Padfield PL, Hodsman GP, Morton JJ. Demeclocycline in the treatment of the syndrome of inappropriate antidiuretic hormone release: with measurement of plasma ADH. Postgrad Med J. 1978;54(635):623-627.

41. Verbalis JG, Ellison H, Hobart M, et al. Tolvaptan and neurocognitive function in mild to moderate chronic hyponatremia: a randomized trial (INSIGHT). Am J Kidney Dis. 2016;67(6):893-901.
42. Saito T, Ishikawa S, Abe K, et al. Acute aquaresis by the nonpeptide arginine vasopressin (AVP) antagonist OPC-31260 improves hyponatremia in patients with syndrome of inappropriate secretion of antidiuretic hormone (SIADH). J Clin Endocrinol Metab. 1997;82(4): 1054-1057.

43. Shoaf SE, Bramer SL, Bricmont P, Zimmer CA. Pharmacokinetic and pharmacodynamic interaction between tolvaptan, a non-peptide AVP antagonist, and furosemide or hydrochlorothiazide. J Cardiovasc Pharmacol. 2007;50(2):213-222.

44. Sahay M, Sahay R. Hyponatremia: a practical approach. Indian JEndocrinol Metab. 2014;18(6):760-771.

45. Peri A, Giuliani C. Management of euvolemic hyponatremia attributed to SIADH in the hospital setting. Minerva Endocrinol. 2014;39(1):33-41.

46. Bordi P, Tiseo M, Buti S, Regolisti G, Ardizzoni A. Efficacy and safety of long-term tolvaptan treatment in a patient with SCLC and SIADH. Tumori. 2015;101(2):e51-e53.

47. Samsca ${ }^{\circledR}$ [package insert]. Rockville, MD: Otsuka America Pharmaceutical, Inc.; Princeton, NJ; 2009.

48. Torres VE, Higashihara E, Devuyst O, et al. Effect of tolvaptan in autosomal dominant polycystic kidney disease by CKD stage: results from the TEMPO 3:4 trial. Clin J Am Soc Nephrol. 2016;11(5):803-811.

49. King JD, Rosner MH. Osmotic demyelination syndrome. Am J Med Sci. 2010;339(6):561-567.

50. Shoaf SE, Bricmont P, Mallikaarjun S. Effects of CYP3A4 inhibition and induction on the pharmacokinetics and pharmacodynamics of tolvaptan, a non-peptide AVP antagonist in healthy subjects. BrJ Clin Pharmacol. 2012;73(4):579-587.

51. Sood L, Sterns RH, Hix JK, Silver SM, Chen L. Hypertonic saline and desmopressin: a simple strategy for safe correction of severe hyponatremia. Am J Kidney Dis. 2013;61(4):571-578.

52. Verbalis JG, Adler S, Schrier RW, et al. Efficacy and safety of oral tolvaptan therapy in patients with the syndrome of inappropriate antidiuretic hormone secretion. Eur J Endocrinol. 2011;164(5):725-732.

53. Berl T, Quittnat-Pelletier F, Verbalis JG, et al. Oral tolvaptan is safe and effective in chronic hyponatremia. J Am Soc Nephrol. 2010;21(4): 705-712.

54. Salahudeen AK, Ali N, George M, Lahoti A, Palla S. Tolvaptan in hospitalized cancer patients with hyponatremia: a double-blind, randomized, placebo-controlled clinical trial on efficacy and safety. Cancer. 2014; 120(5):744-751.

55. Kenz S, Haas CS, Werth SC, Bohnet S, Brabant G. High sensitivity to tolvaptan in paraneoplastic syndrome of inappropriate $\mathrm{ADH}$ secretion (SIADH). Ann Oncol. 2011;22(12): 2696.

56. Berardi R, Caramanti M, Fiordoliva I, et al. Hyponatraemia is a predictor of clinical outcome for malignant pleural mesothelioma. Support Care Cancer. 2015;23(3):621-626.

57. De Las Peas R, Ponce S, Henao F, et al. SIADH-related hyponatremia in hospital day care units: clinical experience and management with tolvaptan. Support Care Cancer. 2016;24(1):499-507.

58. Schrier RW, Gross P, Gheorghiade M, et al. Tolvaptan, a selective oral vasopressin V2-receptor antagonist, for hyponatremia. N Engl J Med. 2006; 355(20):2099-2112.
Cancer Management and Research

\section{Publish your work in this journal}

Cancer Management and Research is an international, peer-reviewed open access journal focusing on cancer research and the optimal use of preventative and integrated treatment interventions to achieve improved outcomes, enhanced survival and quality of life for the cancer patient. The manuscript management system is completely online and includes

\section{Dovepress}

a very quick and fair peer-review system, which is all easy to use. Visit $\mathrm{http}: / /$ www.dovepress.com/testimonials.php to read real quotes from published authors. 